\title{
INFLUENCE OF ACUTE CORONARY SYNDROME ON INTRAOPERATIVE DYNAMICS OF IL-6 DURING CORONARY REVASCULARIZATION
}

\author{
Dmytro Dziuba \\ Department of anesthesiology and intensive care ${ }^{1}$ \\ dr_dzuba@ukr.net \\ Serhii Veremchuk \\ Department of anesthesiology and intensive care ${ }^{1}$ \\ Feofaniya Clinical Hospital of State Management of Affairs of Ukraine \\ 21 Ac. Zabolotnogo str. 21, Kiyv, Ukraine, 03143 \\ veremchuks@ukr.net \\ Oleh Loskutov \\ Department of anesthesiology and intensive care ${ }^{1}$ \\ 5 a Bratislavska str., Kiyv, Ukraine, 02660 \\ doclosk@gmail.com \\ ${ }^{1}$ Shupyk National Medical Academy of Postgraduate Education \\ 9 Dorohozhytska str., Kiyv, Ukraine, 04112 \\ State Institution "Heart Institute of the Ministry of health of Ukraine"
}

\begin{abstract}
Percutaneous x-ray endovascular angioplasty is one of the most modern methods of treatment of severe forms of cardiovascular diseases. In the pathogenesis of acute coronary syndrome, a special place is occupied by the proinflammatory reaction of the body. The main "secondary" marker is interlekin- 6 . The peak of its production occurs on the first day of damage and correlates with its size.

The aim of the study. To investigate the dynamics of interleukin- 6 level in the perioperative period of coronary revascularization, depending on the presence of acute coronary syndrome.

Materials and methods. This study included 68 patients with coronary artery disease. The patients were divided into three study groups. Group 1 and 2 patients were diagnosed with ACS, and they underwent emergency stenting of coronary vessels in order to recanalize occlusion of the coronary arteries. 3rd group patients did not have ACS and they were recanalized as planned.

Results. Analyzing the results of the study, we found that the highest obtained values of IL-6 were in group 1 in comparison with the group of patients who were surgically treated as planned and after stent placement, the interleukin values were $24.8 \%$ higher in group 1 than in group 2 and almost 4 times higher in group 3 ( $p<0.001)$. When comparing groups with acute coronary syndrome, interleukin- 6 indices were significantly higher by $47.4 \%$ before surgery in group 3 and by $24.9 \%$ after stent placement, which once again confirms the severity of patients with acute myocardial infarction.

Conclusions. Intraoperatively proinflammatory interlekin- 6 after coronary artery stenting tends to increase in groups with acute coronary syndrome, while in the group with stable angina, these indicators are significantly higher by $22.8 \%$ $(p<0.001)$. The dynamics of Il-6 levels clearly correlates with the severity of the patient's condition and increases in the presence of acute coronary syndrome, especially in the presence of acute myocardial infarction.
\end{abstract}

Keywords: coronary artery stenting, interlekin-6, acute coronary syndrome.

DOI: $10.21303 / 2504-5679.2020 .001413$

\section{Introduction}

In recent years, cardiovascular diseases (CVD) have occupied a disappointing position as the leading cause of death worldwide. Thus, $45 \%$ of all causes of death in Europe and the United States are attributed to them. More than 4 million people die each year from coronary heart disease (CHD) and strokes. Even more require hospitalization and long-term treatment. Direct and non-direct economic losses of the EU amount to more than 210 billion euros annually [1-5]. According to the report 
of the American Heart Association (AHA) for 2020, $7.4 \%$ of men and $6.2 \%$ of women suffer from $\mathrm{CHD}$, and myocardial infarction occurs in $3 \%$ of citizens over the age of 20 . Every year in the United States, acute myocardial infarction (AMI) occurs in 605,000 patients, and repeated in 200,000. The average age of AMI in men is 65.6 years, in women is 72 years [6-8]. About $\$ 1.1$ trillion is spent annually on CVD treatment in the United States. More than 8 million people have CVD in Ukraine, which puts these diseases in the first place, as well as around the world, with 12,000 of them having AMI [9], and the total mortality from CHD in 2015 was 433 per 100,000 population [1, 3, 4].

In the pathogenesis of acute coronary syndrome, a special place is occupied by the proinflammatory reaction of the body. During hypoxia, the integrity of endothelial cells deteriorates, as well as their barrier function suffers. Vascular permeability increases and white blood cell infiltration increases. When the ischemic period is prolonged, the mechanisms of parenchymal and cardiomyocyte cell death are activated by necrosis, and then apoptosis and autopsy are added for a second time. Necrosis and damage to heart cells and damage to the extracellular matrix release substances that play the role of danger signals that initiate the immune system to protect parenchyma cells and through infiltration of white blood cells contribute to the powerful activation of pro-inflammatory mediators. Pro-inflammatory cytokines, chemokines, and cell adhesion molecules play this role [10].

The key pro-inflammatory cytokines during myocardial ischemia are Il- $1_{\beta}, \mathrm{Il}-6$ and $\mathrm{TNF}_{\alpha}$. Il-1 is a precursor of pro-inflammatory cytokines, and is called "endogenous pyrogen" for its ability to provoke fever. One of its forms is Il- $1_{\beta}$, activated by caspase-1. It has a short half-life and is determined within the first two hours after MI and correlates with the volume of myocardial damage. The main "secondary" marker is Il-6. It is produced in response to a number of stimuli, one of which is Il-1. The peak of its production occurs on the first day of damage and correlates with its size. Another pro-inflammatory agent is $\mathrm{TNF}_{\mathrm{a}}$ It is released by cells during inflammation and correlates not only with the level of myocardial damage, but also with the systolic function of the heart. High levels of these cytokines are closely associated with lethality [11-15].

Percutaneous x-ray endovascular angioplasty is one of the most modern methods of treatment of severe forms of CHD. More than half a million of these interventions are performed annually in the United States [6]. It is during these procedures that the study of the dynamics of pro-inflammatory cytokines is of particular scientific interest. Because, despite the development of $\mathrm{x}$-ray endovascular surgery, the percentage of complications remains quite high, and this is due to the processes of peri-operative inflammation to no small degree, and their determination can affect both the definition of risk and the strategy of treatment of CHD.

The aim of the study. To investigate the dynamics of interleukin- 6 level in the perioperative period of coronary revascularization, depending on the presence of acute coronary syndrome.

\section{Materials and methods of the study.}

This study was conducted in the period from September 2018 to March 2019 on the basis of the State Institution "Heart Institute of the Ministry of Health of Ukraine" and Feofaniya Clinical Hospital of State Management of Affairs of Ukraine. It consisted of 68 patients with coronary artery disease.

The patients were divided into three study groups. Group 1 and 2 patients were diagnosed with acute coronary syndrome (ACS), and they underwent emergency stenting of coronary vessels in order to recanalize occlusion of the coronary arteries. 3rd group patients did not have ACS and they were recanalized as planned.

This study was conducted according to the conclusion of the ethics committee of P. L. Shupyk National Medical Academy of Postgraduate Education (Prot. \#10 from 20/11/2017). During the experiment, bioethical principles, legislation and biomedical research requirements, namely, the Declaration of Helsinki (1964-2013), the Constitution of Ukraine and the Civil Code of Ukraine (2006), the Basics of the Legislation of Ukraine on Health Care (1992), and the Guidelines of Clinical Research of the Ministry of Health of Ukraine No. 42-7.0: 2005 "Medicines. Good Clinical Practice" (2005), Model Regulations on Ethics Commissions for Clinical Trials (Or-der of the Ministry of Health of Ukraine No. 690 of 23-9.2009), Law of Ukraine No. 3447-IV “On protection of animals from ill-treatment" (2006) were observed. 
23 patients were diagnosed with acute myocardial infarction (AMI) (18 men and 5 women, aged 61.3-82.1, on average 69.0 \pm 7.4 years) and they formed the first group of the study. 20 cases with a diagnosis of unstable angina (UA) (15 men and 5 women aged 61.7-77.2, on average 68.3 \pm \pm 6.9 years) made up the second group of the study. The third group of the study included 25 patients diagnosed with stable angina pectoris and underwent planned coronary artery stenting (19 men and 6 women aged 52.6-74.8, on average $66.4 \pm 7.3$ years).

The diagnosis of AMI was made on the basis of clinical data (intensive angina attack was not stopped for more than 30 minutes); ECG data (pathological q wave, St segment elevation by at least $2 \mathrm{~mm}$ from the isoline or its depression by more than $2 \mathrm{~mm}$, inversion of the $\mathrm{t}$ wave); indicators of clinical blood analysis: leukocytosis, increased serum enzyme levels (troponin I, CPK-MB, $\mathrm{ACT}, \mathrm{LDH}$ ); heart ultrasound data (detection of akinesia zones). The average time of admission of patients to the department from the onset of AMI symptoms was $115.4 \pm 37.4$ minutes The area of the left ventricular myocardium lesion when calculating the QRS index was on average $20.4 \pm 1.2 \%$. In 7 (30.4\% of cases) patients, symptoms of cardiogenic shock were observed

The criteria for exclusion from the study were the following: progressive kidney failure, liver dysfunction, acute and chronic infection, heart failure, surgery and trauma for 3 months, inflammation, anemia, peripheral vascular disease, suspected systemic thrombotic diseases and pregnancy, diabetes and cancer, other heart diseases, thyroid dysfunction and autoimmune diseases.

During the study, we detected IL-6 levels before surgery and 10 minutes after stent implantation. During the study, three millilitres of venous blood were collected from the examined patients, and for $30 \mathrm{~min}$. all blood samples were centrifuged at $4^{\circ} \mathrm{C}$ and $3000 \mathrm{rpm}$. within 10 minutes. The serum was separated and stored at $-70{ }^{\circ} \mathrm{C}$ for study.

IL-6 concentrations were determined after collecting all samples, while simultaneously detecting lipidograms, blood glucose and other related indicators. The concentration of serum IL-6 (sensitivity $2 \mathrm{pg} / \mathrm{ml}$ ) was determined by ELISA (Bio-Source Europe S. A., Nivelles, Belgium).

The measurement data in the groups were compared using the Student's t-test or variation analysis; the $\chi^{2}$ test was used to compare the data. Spearman's correlation analysis was used to evaluate correlations. All data was analyzed using SPSS-6.0 software.

\section{Results}

When comparing anthropometric indicators, we found no difference between the study groups by age, gender, and body mass index (BMI). In the laboratory, the total cholesterol, triglycerides, and lipoproteins also did not show a significant difference $(\mathrm{p}>0.1)$. However, there was a significantly higher level of arterial hypertension in group 3. This is shown in Table $\mathbf{1}$.

Table 1

General characteristics of the study groups $(\mathrm{N}=68)$

\begin{tabular}{cccc}
\hline Indicator & \multicolumn{1}{c}{ Group } & $\mathbf{3}(\mathbf{n = 2 5})$ \\
\cline { 2 - 4 } & $\mathbf{1}(\mathbf{n = 2 3})$ & $\mathbf{2}(\mathbf{n = 2 0})$ & $66.4 \pm 7.3$ \\
\hline Age (years) & $69.0 \pm 7.4$ & $68.3 \pm 6.9$ & $19 / 6$ \\
Gender (male/female) & $18 / 5$ & $15 / 5$ & $26.7 \pm 3.4$ \\
BMI (kg/m2) & $24.8 \pm 3.2$ & $25.1 \pm 2.7$ & $18(72.0 \%)^{*}$ \\
AH (n (\%)) & $13(56.5 \%)$ & $11(55.0 \%)$ & $6.2 \pm 1.1$ \\
TC (mmol/l) & $6.5 \pm 1.3$ & $6.3 \pm 1.2$ & $2.0 \pm 0.7$ \\
TG (mmol/l) & $2.3 \pm 0.8$ & $2.2 \pm 0.6$ & $3.3 \pm 0.6$ \\
LDL (mmol/l) & $3.5 \pm 0.7$ & $3.3 \pm 0.8$ & $1.1 \pm 0.5$
\end{tabular}

Note: $*$ - p<0.001-compared to the control group; $C G$ - control group; SA - stable angina; NS - unstable angina; AMI - acute myocardial infarction; BMI - body mass index; AH-arterial hypertension; TC-total cholesterol; TG-triglycerides; $L D L-l o w-$ density lipoproteins; HDL-high-density lipoproteins 
However, the main goal of our study was to investigate the peri-operative level of interlekin- 6 between the study groups. The results of the study are shown in Table 2.

Table 2

Dynamics of IL-6 in the study groups during the first hour after coronary artery stenting

\begin{tabular}{ccc}
\hline Group & \multicolumn{2}{c}{ IL-6 $(\mathbf{p g} / \mathbf{m l})$} \\
\cline { 2 - 3 } & before PCI & in 10 min \\
\hline Group 1 $(\mathrm{n}=23)$ & $23.17 \pm 0.38$ & $24.1 \pm 1.37$ \\
Group 2 $(\mathrm{n}=20)$ & $15.72 \pm 0.44$ & $19.3 \pm 1.26^{1}$ \\
Group 3 $(\mathrm{n}=25)$ & $6.89 \pm 0.57$ & $6.16 \pm 0.84$
\end{tabular}

Note: $p<0.001$ compared to previous indicators; $P C I-$ percutaneous coronary intervention

When analyzing pro-inflammatory interlekin-6, we found that when compared to the operational level and indicators after coronary artery stenting, a significant increase was observed only in group 2 and was higher by $22.8 \%(\mathrm{p}<0.001)$. The same trend continued in the group with AMI, but in patients with planned stenting, this indicator decreased on the contrary.

When comparing the groups, we noticed that the highest obtained values of IL-6 were in group 1 in comparison with the group of patients who were surgically treated as planned (they were increased by $70.3 \%,(p<0.001)$ and exceeded the level of IL-6 in patients with unstable angina and ACS by an average of $24.8 \%(\mathrm{p}<0.001)$ before surgery. And after stent placement, the interleukin values were $24.8 \%$ higher in group 1 than in group 2 and almost 4 times higher in group 3 $(\mathrm{p}<0.001)$. When comparing groups with ACS, interleukin-6 indices were significantly higher by $47.4 \%$ before surgery in group 3 ( $p<0.001)$, and by $24.9 \%$ after stent placement, which once again confirms the severity of patients with acute myocardial infarction.

\section{Discussion}

Studies in recent years had shown that inflammation plays a key role in the pathogenesis of CHD. Based on them, it is clear that t-cell cytokines release a significant amount of interlekin-6 into the bloodstream during decompensation of this disease. Its concentration is not only a diagnostic, but also a predictive factor. This indicator is a predictor of fatal consequences from cardiac causes. Its role as an agent, which makes it possible to predict lethality, is due not only to its anti-inflammatory or procoagulant properties [16-19], but also to the fact that the polymorphism of the $174 \mathrm{G}$ gene $>\mathrm{C}$ and IL6R associated with the metabolism of interleukin- 6 is associated with the risk of mortality from CVD diseases [20, 21].

So in our study, we clearly found that in myocardial infarction, the level of this cytokine significantly (by 70-75\%) exceeds its level in subcompensated cases. After all, it is associated with the main extra- and intra- hospital lethality.

Even in the analysis of patients with ischemic heart disease with and without ACS, the difference in the level of the cytokine under study is clearly differentiated. Before surgery, the level of Il-6 was 2.1 times higher in group 2. It is also noteworthy that in the presence of a decompensated situation, the proinflammatory response tends to increase, especially in unstable angina, in which after stent placement, the indicators are significantly higher than the presurgical level by $22.8 \%(\mathrm{p}<0.001)$. These data once again emphasize the need for urgent surgical interventions in the presence of GCS.

Also, it should be noted that the dynamics of Il-6 level clearly correlates with the severity of the patient's condition and increases from $6.89 \pm 0.57 \mathrm{pg} / \mathrm{ml}$ in patients without ACS to $23.17 \pm$ $\pm 0.38 \mathrm{pg} / \mathrm{ml}$ in the presence of a myocardial infarction in the evaluation before surgery. This pattern persists even after surgery, which confirms the predictive value of this indicator (Fig. 1).

Study limitations. A limitation of our study is the of interleukin-6 level in coronary revascularization perioperative period.

Prospects for further research. To investigate interleukin-6 level in coronary revascularization after operative and perioperative period depending of comorbidities. 


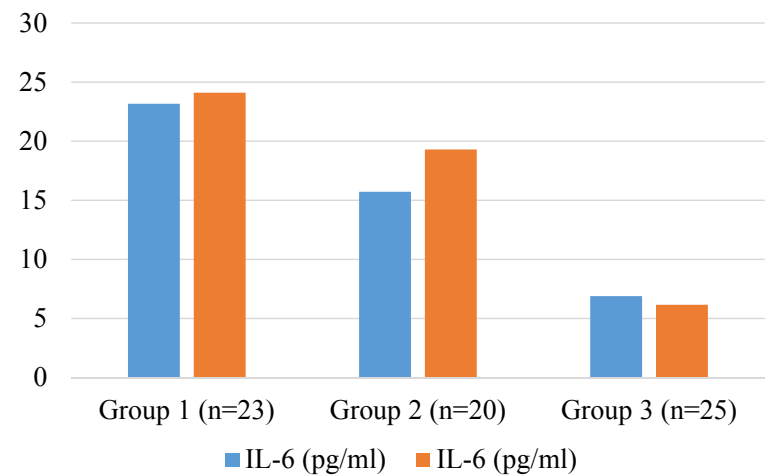

Fig. 1. The level of interleukin- 6 depending on the severity of the patient's condition: blue - level of Il- 6 before stenting; orange - level of Il- 6 after stenting

\section{Conclusions}

1. Intraoperatively proinflammatory interlekin- 6 after coronary artery stenting tends to increase in groups with acute coronary syndrome, while in the group with stable angina, these indicators are significantly higher by $22.8 \%(\mathrm{p}<0.001)$.

2. The dynamics of Il-6 levels clearly correlates with the severity of the patient's condition and increases in the presence of acute coronary syndrome, especially in the presence of acute myocardial infarction.

\section{Conflict of interests}

The authors declare that they have no conflicts of interest.

\section{References}

[1] Movsisyan, N. K., Vinciguerra, M., Medina-Inojosa, J. R., Lopez-Jimenez, F. (2020). Cardiovascular Diseases in Central and Eastern Europe: A Call for More Surveillance and Evidence-Based Health Promotion. Annals of Global Health, 86 (1). doi: http://doi.org/10.5334/aogh.2713

[2] Benjamin, E. J., Virani, S. S., Callaway, C. W., Chamberlain, A. M., Chang, A. R., Cheng, S. et. al. (2018). Heart Disease and Stroke Statistics-2018 Update: A Report From the American Heart Association. Circulation, 137 (12), 67-492. doi: http:// doi.org/10.1161/cir.0000000000000573

[3] Mehta, N. K., Abrams, L. R., Myrskylä, M. (2020). US life expectancy stalls due to cardiovascular disease, not drug deaths. Proceedings of the National Academy of Sciences, 117 (13), 6998-7000. doi: http://doi.org/10.1073/pnas.1920391117

[4] Bhupathi, V., Mazariegos, M., Cruz Rodriguez, J. B., Deoker, A. (2020). Dairy Intake and Risk of Cardiovascular Disease. Current Cardiology Reports, 22 (3). doi: http://doi.org/10.1007/s11886-020-1263-0

[5] Jones, J., Furze, G., Buckley, J. (2020). Cardiovascular Disease Prevention and Rehabilitation. Cardiovascular Prevention and Rehabilitation in Practice, 1-19. doi: http://doi.org/10.1002/9781119071006.ch1

[6] Virani, S. S., Alonso, A., Benjamin, E. J., Bittencourt, M. S., Callaway, C. W., Carson, A. P. et. al. (2020). Heart disease and stroke statistics - 2020 update: a report from the American Heart Association. Circulation, E139-E596.

[7] Gaziano, T. A., Pandya, A., Sy, S., Jardim, T. V., Ogden, J. M., Rodgers, A., Weinstein, M. C. (2019). Modeling the cost effectiveness and budgetary impact of Polypills for secondary prevention of cardiovascular disease in the United States. American Heart Journal, 214, 77-87. doi: http://doi.org/10.1016/j.ahj.2019.04.020

[8] Santos, J. V., Vandenberghe, D., Lobo, M., Freitas, A. (2020). Cost of cardiovascular disease prevention: towards economic evaluations in prevention programs. Annals of Translational Medicine, 8 (7), 512. doi: http://doi.org/10.21037/atm. 2020.01.20

[9] Kovalenko, V. M., Kornatskiy, V. M. (Eds.) (2018). Actual health problems and their minimization in the context of armed conflict in Ukraine. Kyiv: SPD FO «Kolomitsyn VYu», 214.

[10] Prabhu, S. D., Frangogiannis, N. G. (2016). The Biological Basis for Cardiac Repair After Myocardial Infarction. Circulation Research, 119 (1), 91-112. doi: http://doi.org/10.1161/circresaha.116.303577

[11] Seropian, I. M., Sonnino, C., Van Tassell, B. W., Biasucci, L. M., Abbate, A. (2015). Inflammatory markers in ST-elevation acute myocardial infarction. European Heart Journal: Acute Cardiovascular Care, 5 (4), 382-395. doi: http://doi.org/ $10.1177 / 2048872615568965$ 
[12] Mourouzis, K., Oikonomou, E., Siasos, G., Tsalamadris, S., Vogiatzi, G., Antonopoulos, A. et. al. (2020). Proinflammatory Cytokines in Acute Coronary Syndromes. Current Pharmaceutical Design, 26. doi: http://doi.org/10.2174/1381612826666200 413082353

[13] Zeng, C., Wang, R., Tan, H. (2019). Role of Pyroptosis in Cardiovascular Diseases and its Therapeutic Implications. International Journal of Biological Sciences, 15 (7), 1345-1357. doi: http://doi.org/10.7150/ijbs.33568

[14] Jou, C., Shah, R., Figueroa, A., Patel, J. K. (2018). The Role of Inflammatory Cytokines in Cardiac Arrest. Journal of Intensive Care Medicine, 35 (3), 219-224. doi: http://doi.org/10.1177/0885066618817518

[15] Francis Stuart, S. D., De Jesus, N. M., Lindsey, M. L., Ripplinger, C. M. (2016). The crossroads of inflammation, fibrosis, and arrhythmia following myocardial infarction. Journal of Molecular and Cellular Cardiology, 91, 114-122. doi: http:// doi.org/10.1016/j.yjmcc.2015.12.024

[16] Walter, J., Tanglay, Y., du Fay de Lavallaz, J., Strebel, I., Boeddinghaus, J., Twerenbold, R. et. al. (2019). Clinical utility of circulating interleukin- 6 concentrations in the detection of functionally relevant coronary artery disease. International Journal of Cardiology, 275, 20-25. doi: http://doi.org/10.1016/j.ijcard.2018.10.029

[17] Ashiq, S. et. al. (2020). Prevalence and role of different risk factors with emphasis on genetics in development of pathophysiology of coronary artery disease (cad). Pakistan Heart Journal, 52 (4).

[18] Moore, K. J. (2018). Targeting inflammation in CVD: advances and challenges. Nature Reviews Cardiology, 16 (2), $74-75$. doi: http://doi.org/10.1038/s41569-018-0144-3

[19] Vaidya, K., Martínez, G., Patel, S. (2019). The Role of Colchicine in Acute Coronary Syndromes. Clinical Therapeutics, 41 (1), 11-20. doi: http://doi.org/10.1016/j.clinthera.2018.07.023

[20] González-Castro, T. B., Hernández-Díaz, Y., Pérez-Hernández, N., Tovilla-Zárate, C. A., Juárez-Rojop, I. E., López-Narvaez, M. L. et. al. (2019). Interleukin 6 (rs1800795) gene polymorphism is associated with cardiovascular diseases: a meta-analysis of 74 studies with 86,229 subjects. EXCLI journal, 18, 331-355. doi: http://doi.org/10.17179/excli2019-1248

[21] Hansen, P. R., Nelveg-Kristensen, K. E., Rasmussen, H. B., Torp-Pedersen, C., Køber, L., Nielsen, C. H., Enevold, C. (2019). Prognostic role of genetic polymorphisms of the interleukin-6 signaling pathway in patients with severe heart failure. The Pharmacogenomics Journal, 19 (5), 428-437. doi: http://doi.org/10.1038/s41397-019-0068-2 